\title{
A Violation of the Special Theory of Relativity Demonstrated Using the Correlation between Two Pair-Generated Photons
}

\author{
Koshun Suto \\ Chudai-Ji Temple, Isesaki, Japan \\ Email: koshun_suto129@mbr.nifty.com
}

How to cite this paper: Suto, K. (2020) A Violation of the Special Theory of Relativity Demonstrated Using the Correlation between Two Pair-Generated Photons. Journal of Applied Mathematics and Physics, 8, 1755-1766.

https://doi.org/10.4236/jamp.2020.89132

Received: August 9, 2020

Accepted: September 5, 2020

Published: September 8, 2020

Copyright $\odot 2020$ by author(s) and Scientific Research Publishing Inc. This work is licensed under the Creative Commons Attribution International License (CC BY 4.0).

http://creativecommons.org/licenses/by/4.0/

\begin{abstract}
When Einstein developed the special theory of relativity (STR), he assumed the principle of relativity, i.e. that all inertial frames are equivalent. Einstein thought it was impossible to differentiate inertial frames into classically stationary frames where light propagates isotropically, and classically moving frames where light propagates anisotropically. However, the author has previously pointed out that classically moving frames have a velocity vector attached, and presented a thought experiment for determining the size of that velocity vector. The author has already shown a violation of the STR, but this paper presents a violation of the STR using different reasoning. More specifically, this paper searches for a coordinate system where light propagates anisotropically. This is done by using the correlation of two photons pair-generated from a photon pair generator. If the existence of such a coordinate system can be ascertained, it will constitute a violation of the STR.
\end{abstract}

\section{Keywords}

Violation of the Spetial Theory of Relativity, Classically Stationary Frames, Classically Moving Frames, Minkowski Diagrams, Correlation, Quantum Entanglement, Velocity Vector

\section{Introduction}

At the end of the $19^{\text {th }}$ century, most physicists were convinced of the existence of ether as a medium that propagates light. Further, they thought ether to be "absolutely stationary".

Michelson and Morley attempted to detect Earth's motion relative to the luminiferous ether, i.e. the absolute velocity. However, they failed to detect the ef- 
fect they had expected [1]. In order to explain why they failed to detect the effect they had expected, Michelson concluded that the ether was at rest relative to the earth in motion (i.e. it accompanied the earth).

On the other hand, Lorentz was convinced of the earth's motion relative to the "preferred frame". He made a stopgap solution by proposing a hypothesis that a body moves through space at the velocity $\boldsymbol{v}$ relative to the ether contracted by a factor of $\sqrt{1-(v / c)^{2}}$ in the direction of motion [2].

Michelson believed that light emitted from a laboratory on earth propagated isotropically, while light propagated anisotropically in the interpretation of Lorentz.

However, in his special theory of relativity (STR) published in 1905, Einstein insisted that physics did not require an "absolutely stationary frames" provided with special property, and that there be no such things as "specially-favoured" coordinate systems to occasion the introduction of the ether-idea [3].

As a physical theory representing the $20^{\text {th }}$ century, the STR has held sway in the world of physics for more than a century. During this time, the STR has fended off challenges and counterarguments from many physicists [4].

When Einstein developed the STR, he assumed the principle of relativity, i.e. that all inertial frames are equivalent. Einstein thought it was impossible to differentiate inertial frames into classically stationary frames $S_{\mathrm{cl}}$ where light propagates isotropically, and classically moving frames $S_{\mathrm{cl}}^{\prime}$ where light propagates anisotropically. However, the author has previously pointed out that classically moving frames have a velocity vector attached, and presented a thought experiment for determining the size of that velocity vector [5] [6] [7] [8]. The author has already shown a violation of the STR, but this paper presents a violation of the STR using different reasoning.

The thought experiment discussed here strictly distinguishes between classically stationary frames and classically moving frames.

In the STR, the Cartesian coordinate system and oblique coordinate system of the Minkowski diagram are equivalent, and the two can be interchanged. However, in this paper, the argument is developed by placing classically stationary frames into correspondence with the Cartesian coordinate system of the Minkowski diagram, and placing classically moving frames into correspondence with the oblique coordinate system of that diagram.

Einstein assumed the following two principles when developing the STR.

1) Principle of relativity;

2) Principle of constancy of light speed.

First, Einstein explained the principle of relativity as follows [9].

"The laws by which the states of physical systems undergo change are not affected, whether these changes of state be referred to the one or the other of two systems of coordinates in uniform translatory motion".

In addition, he explained the principle of constancy of light speed as follows [3].

"Light is always propagated in empty space with a definite velocity $c$ which is 
independent of the state of motion of the emitting body".

Light is always propagated at a constant velocity $c$, regardless of the velocity of the source emitting the light. In this paper, this principle is called the "principle of constancy of light speed I" (principle I) (However, note that Einstein himself did not classify the principle of constancy of light speed).

Einstein also said the following [3].

"These two postulates suffice for the attainment of a simple and consistent theory of the electrodynamics of moving bodies based on Maxwell's theory for stationary bodies".

However, the STR cannot be developed with these two assumptions alone (principle of relativity and principle I). Einstein also explained the principle of constancy of light speed as follows [10].

"Let a ray of light start at the 'A time' $t_{\mathrm{A}}$ from $\mathrm{A}$ towards $\mathrm{B}$, let it at the ' $\mathrm{B}$ time' $t_{\mathrm{B}}$ be reflected at $\mathrm{B}$ in the direction of $\mathrm{A}$, and arrive again at $\mathrm{A}$ at the ' $\mathrm{A}$ time' $t_{\mathrm{A}}^{\prime}$.

$$
t_{\mathrm{B}}-t_{\mathrm{A}}=t_{\mathrm{A}}^{\prime}-t_{\mathrm{B}} .
$$

[...]

In agreement with experience we further assume the quantity

$$
\frac{2 \mathrm{AB}}{t_{\mathrm{A}}^{\prime}-t_{\mathrm{A}}}=c,
$$

to be a universal constant-the velocity of light in empty space".

In Formula (2), when the distance covered by light making a round trip over the interval $\mathrm{AB}$ is divided by the time needed for the round trip, light speed becomes $c$. This principle will be called the "principle of constancy of light speed II" (principle II).

Incidentally, even if principle II holds, light on the outward path does not necessarily propagate isotropically. However, if the light source is in a classically stationary frame, then by definition light on the outward path propagates isotropically (In this case, light speed on both the outward and return path is $c$ ).

This principle will be called the "principle of constancy of light speed O" (principle O).

Einstein claimed that all inertial frames are equivalent from the standpoint of the principle of relativity. However, among the coordinate systems regarded as inertial frames, this paper defines a coordinate system in which principle $\mathrm{O}$ holds to be a classically stationary frame. Also, a coordinate system in which principle II holds but $\mathrm{O}$ doesn't is defined to be a classically moving frame (Principle $\mathrm{O}$ is a special case of principle II).

In frame $S_{\mathrm{cl}}$, light propagates isotropically, and in frame $S_{\mathrm{cl}}^{\prime}$, light propagates anisotropically. The reason why light cannot propagate isotropically in frame $S_{\mathrm{cl}}^{\prime}$ is the velocity vector attached to frame $S_{\mathrm{cl}}^{\prime}$ (Appendix).

The author has already presented a thought experiment demonstrating the existence of that velocity vector. The author has also pointed out the contradictions of the STR in another paper [11]-[18]. 
The author has already demonstrated a violation of the STR, but this paper demonstrates a violation of the STR using a different thought experiment that is easier to understand. If a coordinate system in which light propagates anisotropically can be found amount coordinate systems regarded as inertial frames, that will constitute a violation of the STR.

\section{Anisotropic Propagation of Light Demonstrated Using the Correlation between Two Pair-Generated Photons}

In this section, an experiment is conducted in rocket A moving at constant velocity of $0.6 c$ with respect to frame $S_{\mathrm{cl}}$. The $x_{\mathrm{A}}$-axis passing through the center of the room coincides with the direction of motion of rocket $A$, and with the $x$-axis of frame $S_{\mathrm{cl}}$ is maintained.

Now, let's observe, from frame $S_{\mathrm{cl}}$, the propagation of light emitted from a light source on the moving rocket.

The light speed of a moving frame does not depend on velocity of the light source (principle I). Light emitted from the moving frame propagates isotropically with respect to frame $S_{\mathrm{cl}}$.

Therefore, viewing from frame $S_{\mathrm{cl}}$, light arrives first at the rear wall of the rocket before the front wall (However, it is assumed that the distances from the light source to the front and back walls are equal).

This section presents a method for checking, through an experiment in the rocket, that light emitted from inside the rocket is propagated anisotropically.

At the point $x_{\mathrm{A}}=0$ in the rocket, a photon pair generator (light source) is placed enabling emission of two photons linearly polarized in the same direction from a specific atom (Figure 1).

The polarization state of a photon is in can be determined from the probability of the photon passing through a polarizer with determinate axis orientation.

Now, consider the situation of photon 1 and photon 2, pair-generated from the light source, moving in the positive and negative direction of the $x_{\mathrm{A}}$-axis. The slits in polarizer 2 (the horizontal polarizer) in the negative direction of the $x_{\mathrm{A}}$-axis are set parallel with the $y_{\mathrm{A}}$-axis. Also, the slits in polarizer 1 (vertical polarizer, analyzer 1) placed at the point $x_{\mathrm{A}}=-L$ are set parallel to the $z_{\mathrm{A}}$ -axis. The angle formed by the slits of the two polarizers is kept perpendicular.

Photon 1 passes through polarizer 2 with a probability of $1 / 2$. According to quantum mechanics, there is a correlation between the two pair-generated photons, and thus at the moment photon 1 passes through polarizer 2 , the polarization of photon 2 moving in the opposite direction is determined to be horizontal polarization.

The orientation of the photon which has passed through polarizer 2 becomes perpendicular with the slits of polarizer 1, so this photon cannot pass through polarizer 1 . At the moment when the photon is blocked by polarizer 1 , the probability of photon 2, moving in the opposite direction, passing through the vertical polarizer 3 (analyzer 2) becomes zero. 

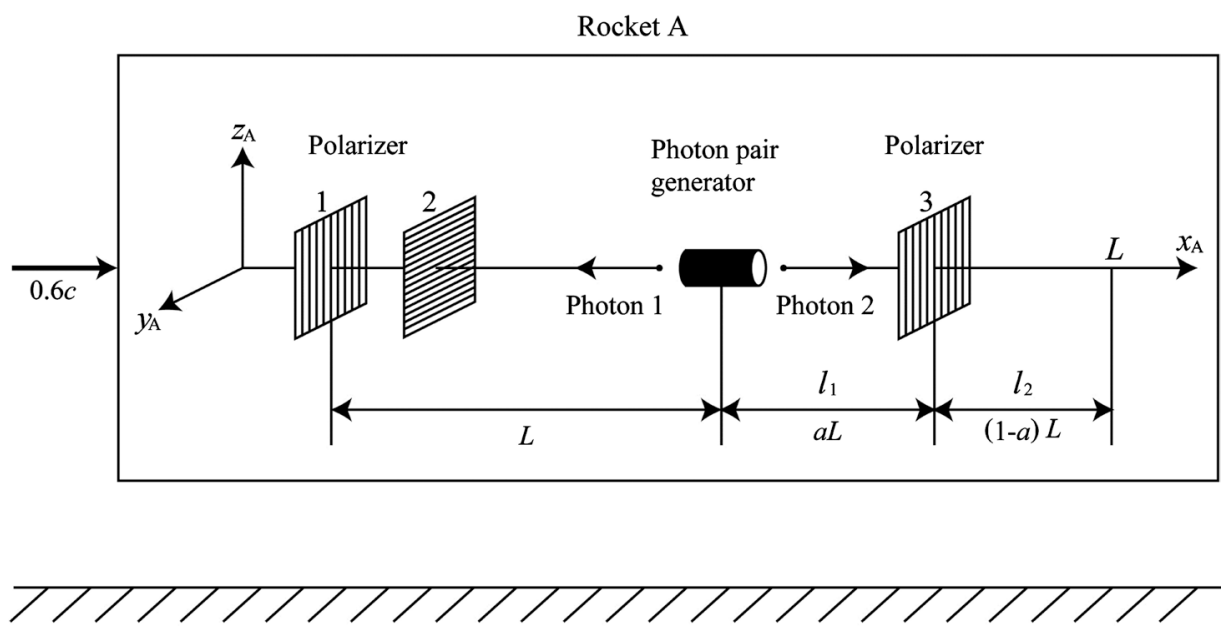

Classically stationary frame

Figure 1. Photon 1 and photon 2 are emitted in opposite directions from the photon pair generator. Polarizer 1 (a vertical polarizer) is placed at the point $x_{\mathrm{A}}=-L$. Also, polarizer 2 (a horizontal polarizer) is placed on the near side of polarizer 1. Polarizer 3 is placed in the positive direction of the $x_{\mathrm{A}}$-axis, and can be moved along the $x_{\mathrm{A}}$-axis. In this paper, this polarizer 3 is moved along the $x_{\mathrm{A}}$-axis. A point (boundary) is found where photon 2, which has passed through polarizer 3 , can no longer pass through polarizer 3 . From the moment when photon 1 is blocked at polarizer 1, photon 2 can no longer pass through polarizer 3 . If this point on the $x_{\mathrm{A}}$-axis can be determined, then the velocity of rocket A can be determined by an experiment in the moving frame. However, this diagram was drawn by shifting the position of polarizer 3 to the right for easier visibility.

Until the moment when the two photons fly out, they are interacting. However, when the two photons have separated, classical thinking holds that there is no influence or action between the two. Einstein thought that way too. However, according to quantum mechanics, even if the two pair-generated photons are far away from each other, they can't completely separate and are in an entangled state (quantum entanglement).

Next, let's discuss this situation using a Minkowski diagram.

The thought experiment discussed here strictly distinguishes between classically stationary frames and classically moving frames.

In the STR, the Cartesian coordinate system and oblique coordinate system of the Minkowski diagrams are equivalent, and the two can be interchanged [19] [20]. However, in this paper, the argument is developed by placing classically stationary frames into correspondence with the Cartesian coordinate system of the Minkowski diagrams, and placing classically moving frames into correspondence with the oblique coordinate system of that diagram (Figure 2).

In this paper, frame $S_{\mathrm{cl}}$ is placed in correspondence with the Cartesian coordinate system of the Minkowski diagram. The $c t$-axis is the world line for $x=0$.

Point $\mathrm{O}$ indicates both origins: $x=0, t=0$ and $x_{\mathrm{A}}^{\prime}=0, t_{\mathrm{A}}^{\prime}=0$. The $x$-axis indicates the $x$-axis of the inertial frame $S_{\mathrm{cl}}$ when $t=0$. In addition, the $x_{\mathrm{A}}^{\prime}$ -axis corresponds to the $x_{\mathrm{A}}$-axis in the rocket at $t_{\mathrm{A}}^{\prime}=0$. The $c t_{\mathrm{A}}^{\prime}$-axis is the 


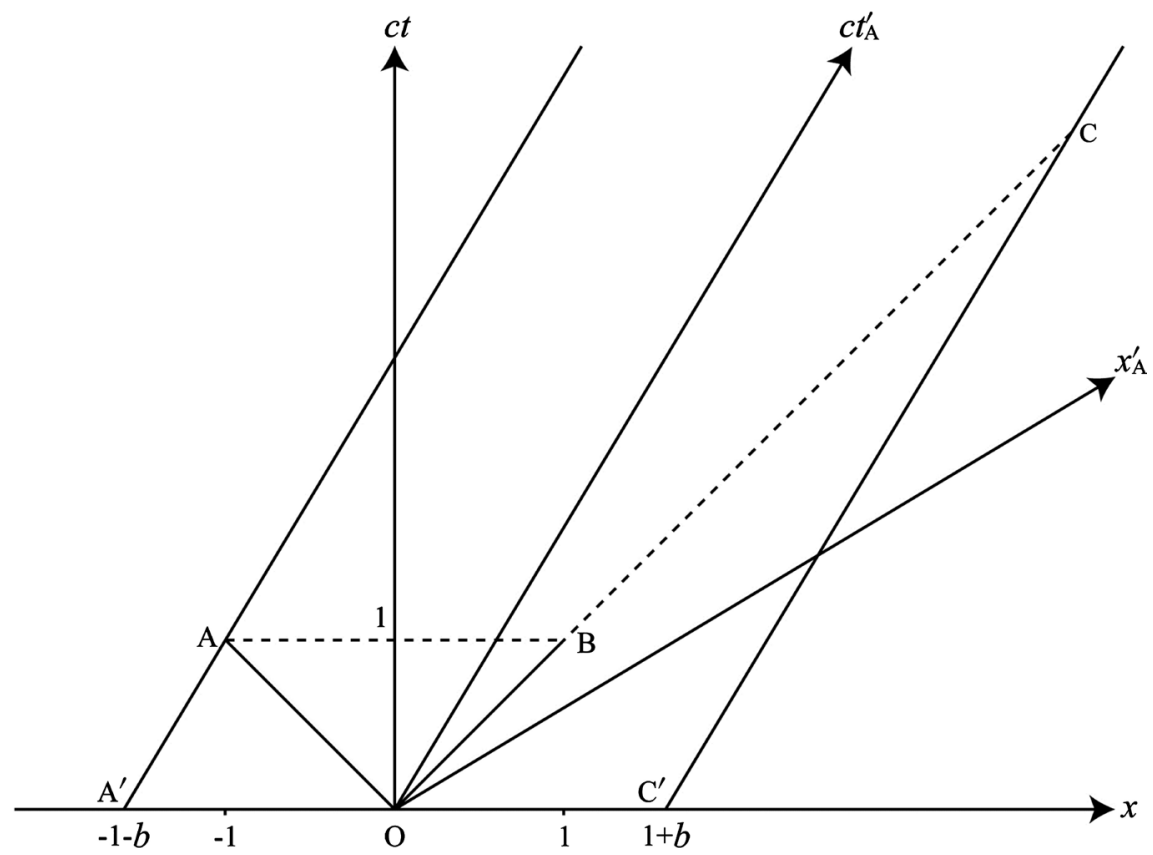

Figure 2. Of the two photons pair-generated from light source $\mathrm{O}$, photon 1 arrives at polarizer 1 at spacetime point A. At this time, photon 2 moving in the opposite direction has arrived at spacetime point $B$. This diagram is for when the rocket velocity is assumed to be $0.6 c$. The events at spacetime points $A$ and $B$ are simultaneous events when seen from frame $S_{\mathrm{cl}}$, and the straight line connecting spacetime points $\mathrm{A}$ and $\mathrm{B}$ is parallel with the $x$-axis. There is also a correlation between photon 1 and photon 2 , and thus at the moment photon 1 can no longer pass through polarizer 1 , photon 2 can no longer pass through polarizer 3 . The point on the spacetime graph where that event occurs is spacetime $\mathrm{B}$. The region where photon 2 has a probability of passing through polarizer 3 is the interval OB $\left(0<x_{\mathrm{A}}<l_{1}\right.$ in terms of $x_{\mathrm{A}}$-coordinates). The prediction of the STR, in contrast, is that the region where photon 2 has a probability of passing through polarizer 3 is the interval OC $\left(0<x_{\mathrm{A}}<L\right)$.

world line of the light source placed at the point $x_{\mathrm{A}}=0$. In addition, the straight line extending at a $45^{\circ}$ angle from $\mathrm{O}$ indicates the light signal emitted from the light source.

In the $x_{\mathrm{A}}^{\prime}-c t_{\mathrm{A}}^{\prime}$ frame, the line parallel to the $x_{\mathrm{A}}^{\prime}$-axis is synchronous in this coordinate system [21].

Spacetime point $\mathrm{A}$ is the spacetime point at the moment when photon 1 has arrived at polarizer 1 (analyzer 1). Also, spacetime point B is the spacetime point of photon 2 at that moment.

The line passing through spacetime points $\mathrm{A}^{\prime}$ and $\mathrm{A}$ is the world line of polarizer 1 placed at the point $x_{\mathrm{A}}=-L$. The line passing through spacetime points $\mathrm{C}^{\prime}$ and $\mathrm{C}$ is the world line for $x_{\mathrm{A}}=L$. These two world lines are parallel to the $c t_{\mathrm{A}}^{\prime}$-axis.

An observer in frame $S_{\mathrm{cl}}$ applies principle I to propagation of photons in a moving frame. According to this principle, the speeds of light emitted from a stationary frame and moving frame are the same, and thus the world line of photon 1 is $\mathrm{OA}$, and the world line of photon 2 is $\mathrm{OB}$. 
For this reason, photon 2 can be propagated over the interval $\mathrm{OB}\left(0<x_{\mathrm{A}}<l_{1}\right.$ along the $x_{\mathrm{A}}$-coordinate) (However, the photon cannot pass through spacetime point B). Photon 2 cannot proceed beyond spacetime point $\mathrm{B}$. The frame $S_{\mathrm{cl}}^{\prime}$ where light propagates anisotropically has this property.

Next, let's decide on a coordinate of the $x_{\mathrm{A}}$-axis corresponding to spacetime point $\mathrm{B}$. If a point in these coordinates is determined, then the velocity of the moving frame can also be calculated. In this paper, that position is determined using two methods.

Method 1. When propagation of light in the rocket is observed from frame $S_{\mathrm{cl}}$, the following formula holds.

$$
\frac{L}{\gamma(c+v)}=\frac{a L}{\gamma(c-v)}, \quad \gamma=\frac{1}{\sqrt{1-v^{2} / c^{2}}} .
$$

Here, $a$ is defined as follows.

$$
a=\frac{l_{1}}{L}, \quad 0<a<1 .
$$

Here, the left side of Formula (3) is the value predicted using a clock in a stationary frame by an observer in frame $S_{\mathrm{cl}}$ of the time required for photon 1 to arrive a polarizer 1 . $a L$ on the right side is the distance $l_{1}$ covered by photon 2 in the positive direction of the $x_{\mathrm{A}}$-axis when that time elapses. The formula contains $\gamma$ because, viewing from frame $S_{\mathrm{cl}}$, the moving object contracts by $1 / \gamma$ times in the direction of motion.

Solving Formula (3), the following values are obtained.

$$
\begin{gathered}
a=\frac{l_{1}}{L}=\frac{c-v}{c+v} . \\
l_{1}=\frac{(c-v) L}{c+v} . \\
v=\frac{(1-a) c}{1+a} .
\end{gathered}
$$

If the value of $a$ is determined by measurement, then the velocity of the moving frame can be calculated from that.

Method 2. Method of finding the position from the Minkowski diagram

First the world lines of the Minkowski diagram and the length along the $x_{\mathrm{A}}$ -coordinate in the laboratory are placed into correspondence as follows.

$$
\mathrm{OB}=l_{1}, \mathrm{BC}=l_{2}, \mathrm{OC}=L .
$$

From this, $a$ on the spacetime diagram is defined as follows.

$$
a=\frac{\mathrm{OB}}{\mathrm{OC}}
$$

In Figure 2, the $x_{\mathrm{A}}^{\prime}$-axis and $c t_{\mathrm{A}}^{\prime}$-axis are given by the following formulas.

$$
c t=b x, \quad b=\frac{v}{c} \text {. }
$$




$$
c t=\frac{1}{b} x .
$$

Here, $b$ is the inclination of the $x_{\mathrm{A}}^{\prime}$-axis.

Also, lines OA and OB for the two light signals can be expressed with the following formulas.

$$
\begin{gathered}
c t=-x . \\
c t=x .
\end{gathered}
$$

Next, the world line for polarizer 1 is the line passing through spacetime points $\mathrm{A}$ and $\mathrm{A}^{\prime}$. The coordinates of the intersection of this line and the $x$-axis are $(-1-b, 0)$. Since the slope of this line is $1 / b$, its equations is

$$
c t=\frac{x}{b}+\frac{1+b}{b} \text {. }
$$

In contrast, the line passing through spacetime points $C$ and $C^{\prime}$ is as follows because the $x$-coordinate of $C^{\prime}$ is $1+b$

$$
c t=\frac{x}{b}-\frac{1+b}{b} \text {. }
$$

Next, the $X$-coordinates of spacetime points A and C are found from these equations.

First, to find the $x$-coordinates of spacetime point A, it is enough to solve the following simultaneous equations for $x$.

$$
\left\{\begin{array}{l}
c t=-x \\
c t=\frac{x}{b}+\frac{1+b}{b} .
\end{array}\right.
$$

If the $x$ found here is taken to be $x_{\mathrm{A}}$,

$$
x_{\mathrm{A}}=-1 \text {. }
$$

Hence the $x$-coordinate $x_{\mathrm{B}}$ of spacetime point B is

$$
x_{\mathrm{B}}=1 \text {. }
$$

Next, the following simultaneous equations are solved to find the $x$-coordinate of spacetime point $\mathrm{C}$.

$$
\left\{\begin{array}{l}
c t=x \\
c t=\frac{x}{b}-\frac{1+b}{b} .
\end{array}\right.
$$

If the $x$ found here is taken to be $x_{\mathrm{C}}$,

$$
x_{\mathrm{C}}=\frac{1+b}{1-b} \text {. }
$$

Hence,

$$
a=\frac{\mathrm{OB}}{\mathrm{OC}}=\frac{x_{\mathrm{B}}}{x_{\mathrm{C}}}=\frac{1-b}{1+b} .
$$

Also, using $v=b c$, 


$$
\begin{gathered}
a=\frac{c-v}{c+v} . \\
b=\frac{1-a}{1+a} . \\
v=\frac{(1-a) c}{1+a} .
\end{gathered}
$$

In the above, it was possible to find a formula for the velocity of the moving frame using two methods.

In the thought experiment of this paper, $v=0.6 c$. Therefore if this is substituted into Formula (5), the following $a$ is obtained.

$$
a=0.25 \text {. }
$$

In the end, photon 2 has a probability of passing through polarizer 3 placed in the positive direction of the $x$-axis when polarizer 3 is placed in this region.

$$
0<x_{\mathrm{A}}<\frac{L}{4} \text {. }
$$

In the STR, in contrast, light propagates isotropically in all inertial frames, and thus photon 2 has a probability of passing through polarizer 3 when polarizer 3 is placed in the following region.

$$
0<x_{\mathrm{A}}<L
$$

When $v=0.6 c$, a difference arises between the predictions of this paper and the STR in the following region.

$$
\frac{L}{4} \leq x_{\mathrm{A}}<L .
$$

The prediction of the STR is that photon 2 has a probability of passing through polarizer 3 placed in the region of Formula (27), but the prediction of this paper is that photon 2 cannot pass through polarizer 3 placed in this region.

\section{Conclusions}

Einstein asserted that, from the standpoint of the principle of relativity, all inertial frames are equivalent. Therefore, Einstein was unable to accept that, within coordinate systems he regarded as inertial frames, there are classically stationary frames in which light propagates isotropically, and classically moving frames in which light propagates anisotropically.

If an experiment exists which can discriminate between these two types of coordinate systems, then the STR will no longer be a correct theory.

This paper has presented, for a second time, a thought experiment enabling discrimination between classically stationary frames and classically moving frames. This means that the STR has been rejected.

The prediction of this paper is that photon 2 will not pass through polarizer 3 in the following region.

$$
\frac{(1-b) L}{1+b} \leq x_{\mathrm{A}}, \quad b=\frac{v}{c}
$$


This differs from the prediction of the STR, which states that photon 2 has a probability of passing through polarizer 3 in the region $0<x_{\mathrm{A}}<L$.

A difference arises between the predictions of this paper and the STR because the STR does not recognize the existence of the velocity vector attached to the frame $S_{\mathrm{cl}}^{\prime}$.

If the coordinate $l_{1}(=a L)$ can be determined by experiment, then it will be possible to find the velocity of the frame $S_{\mathrm{cl}}^{\prime}$ from Formula (7). If the prediction of this paper is correct, then the author will have demonstrated a violation of the STR using two different methods.

\section{Acknowledgements}

I would like to express my thanks to the staff at ACN Translation Services for their translation assistance. Also, I wish to express my gratitude to Mr. H. Shimada for drawing figures.

\section{Conflicts of Interest}

The author declares no conflicts of interest regarding the publication of this paper.

\section{References}

[1] Michelson, A.A. and Morley, E.W. (1887) On the Relative Motion of the Earth and the Luminiferous Ether. American Journal of Science, 34, 333-345. https://doi.org/10.2475/ajs.s3-34.203.333

[2] Lorentz, H.A. (1904) Electromagnetic Phenomena in a System Moving with Any Velocity Smaller than That of Light. KNAW, Proceedings, Vol. 6, Amsterdam, 809-831.

[3] Einstein, A. (1923) The Principle of Relativity. Dover, New York, 38.

[4] Mueller, G.O. (2006) 95 Years of Criticism of the Special Theory of Relativity (1908-2003): The G.O. Mueller Research Project (GOM-Project Relativity). E. Friebe.

[5] Suto, K. (2010) Violation of the Special Theory of Relativity as Proven by Synchronization of Clocks. Physics Essays, 23, 511-519. https://doi.org/10.4006/1.3474836

[6] Suto, K. (2015) Demonstration of the Existence of a Velocity Vector Missing from the Special Theory of Relativity. Physics Essays, 28, 345-351. https://doi.org/10.4006/0836-1398-28.3.345

[7] Suto, K. (2017) Method of Verifying the Existence of Velocity Vectors Excluded by the Special Theory of Relativity. Journal of Physical Mathematics, 4, 258.

[8] Suto, K. (2018) A Breakdown in the Special Theory of Relativity Demonstrated Based on an Elucidation of the Relativity of Time. Applied Physics Research, 10, 38-49. https://doi.org/10.5539/apr.v10n4p38

[9] Einstein, A. (1923) The Principle of Relativity. Dover, New York, 41.

[10] Einstein, A. (1923) The Principle of Relativity. Dover, New York, 40.

[11] Suto, K. (2020) Violation of the Special Theory of Relativity and Elucidation of the Twin Paradox. In: George, T.F., New Insights into Physical Science, Vol. 4, Book Publisher International, London, 100-115.

[12] Suto, K. (2017) A New Problem with the Twin Paradox. Applied Physics Research, 9, 77-86. https://doi.org/10.5539/apr.v9n2p77 
[13] Suto, K. (2018) The Problem of the Twin Paradox Elucidated Based on a Thought Experiment Carried out by Discriminating between a Classically Stationary Frame and Moving Frame. Journal of Physical Mathematics, 9, Article ID: 1000278.

[14] Suto, K. (2016) Elucidation of Time Symmetry Predicted by the Special Theory of Relativity. IOSR Journal of Applied Physics, 8, 20-27.

http://www.iosrjournals.org/iosr-jap/papers/Vol8-issue6/Version-4/D0806042027.pdf

[15] Suto, K. (2016) Thought Experiment Revealing a Contradiction in the Special Theory of Relativity. Applied Physics Research, 8, 70-74. https://doi.org/10.5539/apr.v8n6p70

[16] Suto, K. (2017) An Elucidation of the Symmetry of Length Contraction Predicted by the Special Theory of Relativity. Applied Physics Research, 9, 31-39. https://doi.org/10.5539/apr.v9n3p31

[17] Suto, K. (2014) Velocity Addition Laws which Can Coexist with the Velocity Addition Law in the Special Theory of Relativity. Physics Essays, 27, 191-197. https://doi.org/10.4006/0836-1398-27.2.191

[18] Suto, K. (2014) True Factors Determining the Ratio of Space Contraction and Time Dilation Predicted by the Special Theory of Relativity. Physics Essays, 27, 580-585. https://doi.org/10.4006/0836-1398-27.4.580

[19] Minkowski, H. (1923) The Principle of Relativity. Dover, New York, 78.

[20] Born, M. (1962) Einstein's Theory of Relativity. Dover, New York, 196-200.

[21] French, A.P. (1968) Special Relativity. Norton \& Company, New York, 76. 


\section{Appendix}

The author has already presented a thought experiment enabling confirmation of whether that velocity vector exists. If a velocity vector exists in that thought experiment, it is also possible to determine the size of that vector.

However, if it is assumed that a velocity vector is attached to a certain inertial frame, then we must also discuss the stationary frame. That stationary frame is not something like the center of gravity of the universe, existing at a place separated from moving frames. Also, it is not desirable to revive the hypothetical material of the ether which was actively discussed in the latter half of the $19^{\text {th }}$ century. The author does not support the views of Lorentz or Michelson. The definitions of "velocity vector" and "stationary frame" conceived by the author are as follows.

According to quantum electrodynamics, a vacuum which transmits electrical force is thought to be filled with opposing pairs of virtual particles and antiparticles. The vacuum can transmit light as a wave. Therefore, let us tentatively assume that these virtual particles are the modern day ether.

Also, according to the "uncertainty principle", these virtual particles are constantly fluctuating and not at rest, even when in the lowest energy state.

Here, it is assumed that a vacuum exists even at the deep layer of a single arbitrary point in the space of an inertial frame. Next, vectors are used to indicate the velocities at a certain time of the countless virtual particles which exist at that point in the vacuum, and then those vectors are combined into a single vector (If there is a problem here with the expression "which exist at the point", it can be changed to the more ambiguous expression "which exist in the neighborhood of that point"). This combined vector is taken to be the velocity vector at that point.

Next, a vector is used to indicate the relative velocity between the combined vector and the inertial frame.

If the relative velocity is zero, this inertial frame is determined to be "classically stationary frame".

Conversely, if the relative velocity is not zero, this inertial frame is determined to be "classically moving frame". However, what determines the direction of this vector is convention.

In this paper, the author feels it is best treat this vector as having a starting point in the vacuum and an end point in the inertial frame of physical space.

In this case, the point in the vacuum plays the role of a stationary frame. Also, the $V_{x}$ is the component in the $x$-axis direction of the velocity vector attached to the inertial frame regarded as a problem here. 